\title{
Sweet Anticipation: Music and the Psychology of Expectation by David Huron
}

\author{
Cambridge, MA: MIT Press, 2006
}

\author{
Reviewed by Marcus T. Pearce and Daniel Müllensiefen \\ Goldsmiths, University of London
}

In the glossary to Sweet Anticipation: Music and the Psychology of Expectation by David Huron, the term sweet anticipation is defined as "the positive feelings that arise from conscious thought about some future event". As such, the term constitutes a rather strange choice of title for a book that focuses to a large extent on unconscious processes involved in musical expectation. Presumably, the intention was to place emphasis on "positive feelings" since this account differs from most written by researchers in music cognition in terms of the prominence it gives to music's ability to invoke pleasure. However, Huron's approach is distinctive in other ways. The prominence given to psychological theories, models and methods as tools for understanding music will come as no surprise for those familiar with his field of research. But the emphasis on taking subjective musical experience seriously, modelling music perception as a process of (statistical) learning, examining music from wide range of cultures and understanding cognition in an evolutionary context represent characteristic hallmarks of this work. We shall return to these themes in more detail later on.

In a recent review of Daniel J. Levitin's book This is Your Brain on Music: The Science of a Human Obsession, David Huron notes the advantages brought to a field of study by having a popular champion and laments the fact that, in spite of being in its "golden age", research in music cognition "has not been communicated beyond a small coterie of professionals" (Huron, 2006, p. 5). It seems likely that this was a strong motivating factor for Huron himself in writing Sweet Anticipation. However, in spite of the informal and times rather anecdotal style (which some will find engaging and others irritating), the technical content and length of this book would probably put it beyond the casually interested member of the public. Rather, the intended audience appears to have been individuals with some technical knowledge of fields of research related to (but distinct from) music cognition. These fall into two camps: first (and foremost), the book is written for musicians (including composers) and musicologists (including music theorists); and second, it is hoped that it will be of interest to researchers in cognitive science and (evolutionary) psychology.

Considering the latter group, it is important to note that Huron often bases his arguments on psychological theories and results, and occasionally offers new perspectives based on his own theory (e.g., a new interpretation of the well-established mere exposure effect). He also presents several arguments for the relevance of music as a useful test bed for a general theory of expectation: first, it is more constrained than most everyday instances of expectation; second, musical activities often involve the active manipulation of expectation; and third, detailed records of the stimulus structure exist in the form of scores. Although it is not stated explicitly, it is worth making a further point (dependent on these three) that music potentially offers a source of more ecologically valid stimuli than those usually used in the experimental study of human expectations (e.g., Saffran et al., 1999). Although the book's detailed treatment of music probably requires some musical background, there is generally a clear demarcation between the presentation of the theory and its application to music while the comprehensive glossary and availability on the Internet of audio clips corresponding to all notated figures will help enormously in this regard. 
The primary reason that this book will be of interest to psychologists and cognitive scientists is that it offers a novel theory of expectation that is intended to be general. Huron's evolutionary approach is founded on the observation that the ability to anticipate future events increases the potential of an organism to survive and reproduce. This is partly because accurately predicted events can be perceived, processed and responded to more quickly and efficiently (as a result of biological and psychological preparation) but also because successful prediction of outcomes allows an organism to select actions with the most positive consequences.

The book opens by presenting a novel theory of expectation (the ITPRA theory) which distinguishes five separable components of the cognitive process of expectation. These components are called into action at different stages during the process of perceiving and responding to an event (the outcome of some process). In the pre-outcome phase, the imaginative response involves imagining and evaluating the possible outcomes while the purpose of the tension response is to tailor attention and arousal according to the nature, uncertainty, importance and imminence of anticipated outcomes. The post-outcome phase involves three distinct responses. At the onset of the event, the prediction response evaluates the extent to which the outcome confirms or violates prior expectations while the reaction response constitutes a fast and automatic response to the nature of the event. (According to Huron, evolution has crafted a pessimistic reactive response that plays safe in the first instance by assuming a worst-case scenario). By contrast, the conscious appraisal response consists of a more leisurely, cortically mediated process of consideration and assessment yielding positive and negative reinforcement associated with the outcome.

Much of the book is spent examining the emotions generated by predictive success and failure. The prediction response ensures that the former lead to positive limbic reward while the latter produce a negative limbic penalty in the form of surprise. These emotional consequences are nature's solution to the fundamental problem of motivating an organism to improve its anticipatory skills. Huron argues forcefully that, in the auditory domain, these skills are acquired largely as a result of statistical induction of regularities in the environment. The evolved capacity to learn is a biological solution to the problem of anticipation in highly variable environments (known as the Baldwin effect).

Huron describes in detail the prediction effect whereby positive emotions resulting (via the prediction response) from anticipatory success are misattributed to the stimulus itself, leading to a preference for predictable events. On the other hand, the stress resulting from surprising events, as an indicator of maladaptive anticipatory failure, has two main effects. First, it activates one of three fast, conservative responses: fight, flight or freeze (depending on the perceived severity of the threat and degree of control over the outcome). Second, it provides the cognitive system with feedback on the predictive utility of different competing representations of the environment. Representations that yield accurate predictions will be strengthened and reused while those that do not will atrophy.

How is it then that surprise can be enjoyable even though it is associated with negative emotion due to the prediction effect? The answer, according to Huron, has to do with contrastive valence: contrasts of emotional valence between the different expectation responses. An event that is welcome but unexpected (e.g., the unannounced arrival of an old friend) induces a negative prediction response that increases the positive limbic effect of the reaction or appraisal responses. Even events that are merely innocuous, but unexpected, can lead to positive emotional consequences due to contrastive valence. Other phenomena can also be accounted for in these terms. For example, delaying an anticipated event (e.g., an important meeting is postponed at the last moment) will lead to a negatively valenced tension response that enhances the positive effects of the prediction response when the event finally arrives.

Huron's principal goal in writing Sweet Anticipation, however, is to introduce psychologically-based insights about music to musicians and musicologists. Several chapters are devoted to using the theory of expectation to explain a range of phenomena related to musical structure and music perception. In doing so, Huron combines the results of a large number of studies in music cognition with his own statistical analyses. However, he provides similar arguments to support his explanations of the perception 
of tonality, meter/rhythm, musical surprise and tension, as well as the evolution of individual stylistic approaches to composition. Specifically, all these phenomena are related to involuntary and implicit learning of regularities in the musical culture in which we participate, and how this process allows us to predict future musical events. These predictions may involve explicit musical knowledge, but they also help us to unconsciously and efficiently process predictable events and respond to unpredictable events while we are listening to music.

The study of musical qualia (the subjective experiences associated with musical events) is a defining characteristic of Huron's approach. In discussing tonality, for example, which has a long history of study in music psychology, he argues that these qualia derive from a process of statistical learning. Reporting the results of an unpublished survey, he links the verbally reported qualia of experienced Western musicians for the scale degrees appearing in a major context to their predictability in Western folk songs. Three aspects of statistical learning are discussed in terms of the qualitative experience of scale degrees: first, the zeroth-order frequency (e.g., the lowered supertonic was frequently described as "surprising" or "abrupt"); second, the frequency at phrase- or work-terminating positions (e.g., the tonic and mediant were frequently described as "restful" or "home"); and finally, the flexibility associated with transitions from the scale degree (e.g., the leading-tone and raised dominant were often described as "leading", "pointing", "upward"). Although other categories of adjectives, like mobility or emotions, require more sophisticated interpretation, Huron manages to provide informal but convincing explanations of the correlations between his categories of qualia and statistical properties of the scale degrees. However, the alternative argument that these qualia simply reflect aspects of formal Western music training is raised but is not addressed entirely convincingly. Furthermore, the extent to which the results of these qualia studies generalise beyond trained musicians remains an open question (a general limitation of using the verbal reports of trained musicians in research on music perception).

Just as expectations for the different scale degrees vary according to their statistical occurrence in Western tonal music, listeners also have strong learnt expectations regarding the timing of musical events. Huron argues that the predictability of events in time provides the foundation of our perception of rhythm and metre and suggests a number of sources for this predictability: periodic metric structure; rhythmic motifs (e.g., in dance styles); the regularity of musical phrases; and even the regularity of non-periodic sequences apparent, for example, in Japanese or West-African traditional music. He supports his argument with a range of experimental evidence, ranging from psychological to neuroscientific, demonstrating that events occurring at predictable points in time are more quickly and easily processed by listeners.

Moving up a level from the perception of individual musical elements, Huron describes how predictability and expectedness can be exploited by composers and performing musicians to achieve creative and aesthetic goals (such as humour). Of particular interest is a concise discussion of the distinction between a genre and an individual musical work, which is highly dependent on the musical culture and historic period, and can sometimes be altogether meaningless. Again the description of a musical work is based on the statistical properties of a musical experience that determine the degree to which an object in auditory memory has a unique mental identity. Uniqueness is determined by markers that appear frequently in the experience, but less commonly in other musical experiences, and are presented early in the experience. According to Huron, pitch, rhythm, and lyrics function as uniqueness markers because they provide more combinatorial possibilities than other compositional devices like instrumentation. This quasi-definition of a musical work provides a good example of Huron's attempt to address a range of high-level musical concepts within his statistical approach. However, a comparison with similar discussions in the literature on MIR (e.g., the TF-IDF measure for melodic similarity in Uitdenbogerd, 2002) or statistical text processing (e.g., Salton, 1983) would, once again, be rewarding.

Much of the applied discussion of musical expectation in Sweet Anticipation is based on a reformulation of concepts from music theory within the framework of statistical learning. For example, Huron reviews compositional techniques for creating tension such as suspension notes, delays of cadential for- 
mulae or climax building, in terms of how they reduce or increase predictive uncertainty in the listener. An experienced music theorist might argue that this does not represent a novel insight since counterpoint, harmony, and music theory are often taught using language related to conventions and expectation. However, no-one has worked out the statistics behind this language as meticulously as Huron does here.

Towards the end of the book, Huron demonstrates that even composers who explicitly try to overthrow musical conventions betray in their music an awareness of their audience's expectations, based on statistical induction of regularities in Western music. Again, it is common knowledge that composers like Wagner, Schönberg and Stravinsky have consciously worked against musical conventions. The value of Huron's musicological discussions of examples from modern Western art music lies in the insight that his statistical approach is compatible with more traditional musicological methods although it has the advantage of allowing the analyst to quantify important stylistic features.

The application of the theory to music is illustrated throughout with references to an impressive range of different musics from different cultures. This emphasis on cross-cultural and ethnomusicological diversity reflects, in part, Huron's preference for a learning-based account of the acquisition and application of musical expectation. Aspects of the musical environments that differ between cultures should lead to differences in the expectations exhibited by listeners while those that remain constant ought to be reflected in similarities. However, the statistical approach to cross-cultural comparison requires musical representations to be transcribed as discrete properties of discrete musical events and Huron fails to acknowledge that this may not always be appropriate either to the performance or perception of the music of a given culture. Representational issues also mean that the statistical analyses reported tend to be restricted to monophony, the only exception being the use of annotated chord progressions in J. S. Bach's chorale harmonisations.

As noted above, Sweet Anticipation is written with music scholars and cognitive scientists in mind. For an interdisciplinary field such as music cognition, it would be hard to overstate the importance of forging links with its parent and sibling fields of study and this work represents a useful contribution to the cause. However, several potentially fruitful directions for bridge-building are left unexplored. For example, recent cognitive scientific research on creativity (Boden, 1990; Wiggins, 2006) would seem highly relevant to the discussion of musical representations and creating various kinds of psychological effect with music (e.g., predictability, surprise, tension). Furthermore, Huron's study of musical humour might be well-served by an examination of scientific research on linguistic humour (e.g., Ritchie, 2003) while his emphasis on music's capacity to evoke pleasure suggests the possibility of a useful link between his theory of expectation and research in experimental aesthetics (Berlyne, 1974; North \& Hargreaves, 1997).

Finally, given Huron's emphasis on corpus-based computer analysis, it is surprising to find how little reference is made to recent research in the Music Information Retrieval (MIR) community (Downie, 2003; Orio, 2006). A central theme of the book, for example, is that the induction of regularities by listeners goes beyond the zeroth-order probability estimates postulated by Krumhansl (1990). Where Krumhansl proposes tonal hierarchy (a zeroth-order distribution of scale degrees), for example, Huron adds tonal tendency (a first-order distribution of scale degrees). However, while he suggests that higherorder probabilities are also internalised by listeners, only monogram (zeroth-order) and digram (firstorder) models are actually used in the analyses. But higher-order statistical models have been examined extensively in a range of domains and the effects of varying the order have been studied specifically in the context of melody prediction (e.g., Conklin \& Witten, 1995; Pearce \& Wiggins, 2004). Furthermore, the suggestion that listeners' expectations are based on "some sort of weighted sum" (p. 110) of contributions from different representations (depending on their predictive utility) has been realised and tested in statistical models of melodic pitch structure (e.g., Conklin \& Witten, 1995; Pearce et al., 2005). As another example of potentially fruitful links to MIR research, Huron's statistical approach might benefit from the use of melodic similarity measures (e.g., Hewlett \& Selfridge-Field, 1998, 2004) which 
would allow frequency counts (for themes, motifs and so on) to be based on approximate as well as exact matches. It would be interesting to see whether allowing matching to slight or moderate variations would strengthen the statistical trends or simply introduce noise.

The final chapter of this book presents various implications raised for musicians and composers, musical aesthetics, musicology and ethnomusicology; but, tellingly, no mention is made of the contributions made to music cognition and the psychology of music. However, it is pertinent to ask whether Sweet Anticipation represents a contribution to Huron's own field of research?

The core theoretical and applied arguments are liberally supported by summary discussions of research carried out in Huron's lab at Ohio State University. Indeed this research provides the backbone of the book and music psychologists with only a passing familiarity of Huron's work will find a useful digest and source of pointers for further reading. However, perhaps the most compelling reason to recommend this book to the music psychologist is that it presents a partisan perspective on music cognition in general and musical expectation in particular. Although it provides cogent summaries of key aspects of research in several important areas of music cognition (e.g., tonality, absolute pitch), this is no general text book. Perhaps the most obvious example is Huron's preference for statistical learning as a model of the cognitive mechanisms involved in music perception and his use of probabilistic and information theoretic measures to study these mechanisms. This affinity to the empiricist view of cognition is never explicitly presented as a preference but is clearly evidenced by casual unqualified assertions such as "Of course English grammar is entirely learned" (p. 14) without any acknowledgement of the ferocity with which this issue continues to be debated (e.g., Pinker \& Jackendoff, 2005). It should also be emphasised that this view is by no means unanimously accepted in the field of music cognition research (see e.g., Lerdahl \& Jackendoff, 1983; Narmour, 1990, and empirical research based on these theories). As one colleague put it: "He likes counting, doesn't he?".

The present reviewers are broadly sympathetic to Huron's theoretical stance but readers with a scientific background will be disappointed to find little comparison (theoretical or empirical) with other theories. Likewise little systematic effort is made to falsify the various models and mechanisms proposed instead of merely presenting positive evidence that is consistent with them (or, conversely, postulating mechanisms that are plausible in the light of current research). In discussing what he calls tonal syncopation, for example, Huron draws attention to the fact that ascending major thirds are more likely to appear in Western folksong in strong-to-weak metrical positions while ascending fourths appear more frequently in weak-to-strong metric contexts, and observes that listeners' perception of the metric emphasis implied by diatonic intervals tends to follow this statistical association. This relationship is of considerable interest and is consistent with a theory of statistical learning (it also provides a representative example of of Huron's use of evidence in arguing for statistical learning). However, there is a chicken and egg problem here that is acknowledged but never satisfactorily addressed. One could reasonably argue that expectations are determined by innate properties of the perceptual system and that statistical regularities in music simply reflect an attempt by composers to satisfy those expectations. In practice, most aspects of cognition are likely to be determined by some complex union of nurture and nature. Cross-cultural, developmental and neuroscientific research will form an important part of determining the nature of the union for any given aspect of music perception (see also Justus \& Hutsler, 2005).

In addition, and in spite of an entire chapter on methodology, the overall lack of experimental detail will frustrate those hoping to find a technical account of the experiments conducted and their results: for example, many references are made to information theoretic quantities which are only ever defined qualitatively. Consider, as a case in point, Shannon entropy which is used frequently as a means of measuring uncertainty but is only ever explained intuitively using descriptive examples. In chapter 7 , meanwhile, Huron plots changes of information for different representations of "Pop Goes the Weasel" without explicitly defining information content or stating how the probabilities used in its calculation were estimated. Equally vague references both to Bayesian reasoning and neuroscientific evidence risk 
leaving those who are familiar with these fields unimpressed and those who aren't confused. As a final example, Huron frequently calculates and plots mean values but rarely presents variances, error-bars or $\mathrm{p}$ values for differences between means.

The lack of methodological detail and reluctance to engage in empirical model comparison makes it hard to evaluate the conclusions Huron draws from his empirical analyses. More generally, the speculative, discursive approach suggests a leaning towards the humanities that is also evidenced by the footnote citation style which will frustrate readers used to author/date or numerical referencing more commonly used in scientific writing. Nonetheless, music psychologists will find in this book a somewhat idiosyncratic perspective on music cognition providing a rich source of hypotheses for further study. For this very reason, however, readers without a detailed knowledge of the field should be warned that this book does not necessarily represent the final word on the psychology of musical expectation (nor even a comprehensive contemporary overview). At the other end of the spectrum, there are also several reasons why Sweet Anticipation will be of interest even to those specialists who are familiar with Huron's published output.

First, the book format permits the author to draw together different strands of research and present them afresh in the context of a unified framework. A good example is provided in chapters 5 and 6 which contain an extended discussion of five statistical regularities in music and listeners' sensitivity to each one. Take, for example, pitch proximity: successive pitches in melodies tend to be proximal (Huron, 2001) and listeners expect this to be the case (Aarden, 2003). Huron argues that melodic expectations are influenced by statistical induction of this relatively ubiquitous regularity that arises as a result of physical constraints on changes of frequency for vibrating objects. However, he also stresses the important distinction between regularities existing in actual music and the representation of these regularities by the human cognitive system. Perhaps the most convincing example presented is melodic regression to the mean: the tendency for pitches in the extremes of the pitch range to be followed by more central pitches (von Hippel \& Huron, 2000). The expectations of listeners, however, exhibit post-skip reversal: an approximation to this regularity where large intervals are expected to be followed by a change of registral direction regardless of pitch height (von Hippel, 2002). The important question of why the representations learnt by listeners should diverge from the actual regularities in the musical environment is considered in chapter 7. As discussed earlier, Huron argues that different representations of the musical surface engage in a competition governed by the predictive utility and simplicity of the representation in the current context. Several kinds of simplicity are defined including a preference for low-order and temporally proximal relationships, lower derivative states and ease of binding to sensory events (onsets). Huron argues that while post-skip reversal is a more complex representation than melodic regression in terms of its higher derivative state (it involves pitch interval relationships rather than pitch relationships) this is more than compensated for by its greater simplicity in terms of order and temporal proximity (the calculation of post-skip reversal involves only the last two events while melodic regression involves maintaining a cumulative distribution of all previous pitches in order to calculate the mean pitch).

A second reason that music psychologists will profit from reading Sweet Anticipation is that the argument is illustrated throughout with the results of unpublished studies, computer analyses, informal observations, anecdotes, general impressions and intuitions. Chapter 3, for example, contains the only published report of an experiment (conducted by David Huron, Paul von Hippel and David Harnish) which used a gambling methodology (following Manzara et al., 1992) to estimate the pitch expectations of Balinese and American musicians while listening to a unfamiliar Balinese melody. Elsewhere we learn that the tendency for large intervals to ascend and small intervals to descend (Vos \& Troost, 1989) has been replicated over the years in Huron's laboratory in a wide range of musical cultures beyond those in which it was originally reported. Many points are illustrated even more informally, but often highly effectively (e.g., the discussion of frisson in music or stylistic regularisation over time), by reference to personal experiences, anecdotes and hypothetical situations. 
All this means that, apart from anything else, David Huron's book provides a wealth of fascinating insights amassed throughout 20 years of research in the field. However, the informal style in which the material is presented sometimes makes it difficult to distinguish between the results of rigorous empirical experiment and casual observation. Once again, one has the feeling that this book forms a more useful source of hypotheses than it does of answers. In particular, many of Huron's proposals will need to be empirically tested in an experimental context and his quantitative analyses replicated with different conditions and assumptions (by systematically varying musical contexts, participant's musical training, various aspects of the statistical models and so on). The research stimulated by Huron's book will be a reflection of its importance as a contribution to research in music cognition.

David Huron's overall research programme, as presented in this book, reflects his background and training in music performance, musicology, psychology and computer science. As such, Sweet Anticipation represents the result of many years of dedicated research along distinctive, consistent lines. It is important to acknowledge, for example, that many of the datasets examined were encoded over many years by Huron and his co-workers in an electronic format (the Humdrum syntax including ${ }^{\star}$ kern). Furthermore, the statistical analyses were conducted using the Humdrum toolkit, a free general-purpose software system for music research. Both components of Humdrum were designed and written by Huron himself.

Perhaps the most striking shortcomings of this book relate to the manner in which it identifies and targets its audience. In spite of the conversational style, this is not intended to be pop-science and is probably too specialised for the general reader. Although the intended audience (music scholars and psychologists) will find a wealth of ideas to transfer and apply to their own fields of study, it is to be emphasised that this book does not provide a general point of entry into the field of music cognition. In addition, the applied discussion of specific music examples may prove too detailed for psychologists while musicologists may feel Huron is going over well-trodden territory. Conversely, while the latter group may find the emphasis on statistical analysis oppressive, the former may be irked by the non-scientific presentation of scientific matter. For the music psychologist, however, Sweet Anticipation provides more than just a summary of Huron's published output. Rather, one is guided through a broad range of research from Huron's laboratory woven together in a unified framework that is illustrated by unpublished studies, small-scale computer analyses, informal observation and general intuitions. As such, and perhaps most compellingly, this book contributes a refreshingly distinctive and personal perspective on music cognition in general, and expectation in particular, that should prove a bountiful source of inspiration for future research in the field.

\section{References}

Aarden, B. (2003). Dynamic Melodic Expectancy. Doctoral dissertation, Ohio State University, Columbus, $\mathrm{OH}$.

Berlyne, D. E. (Ed.). (1974). Studies in the New Experimental Aesthetics: Steps Towards an Objective Psychology of Aesthetic Appreciation. Washington: Hemisphere Publishing Co.

Boden, M. A. (1990). The Creative Mind: Myths and Mechanisms. London: Weidenfield and Nicholson.

Conklin, D. \& Witten, I. H. (1995). Multiple viewpoint systems for music prediction. Journal of New Music Research, 24(1), 51-73.

Downie, J. S. (2003). Music information retrieval. In B. Cronin (Ed.), Annual Review of Information Science and Technology, volume 37 (pp. 295-340). Medford, NJ: Information Today. 
Hewlett, W. B. \& Selfridge-Field, E. (Eds.). (1998). Melodic Similarity. Number 11 in Computing in Musicology. Cambridge, MA: CCARH and MIT Press.

Hewlett, W. B. \& Selfridge-Field, E. (Eds.). (2004). Music Query: Methods, Models, and User Studies. Number 13 in Computing in Musicology. Cambridge, MA: CCARH and MIT Press.

Huron, D. (2001). Tone and voice: A derivation of the rules of voice-leading from perceptual principles. Music Perception, 19(1), 1-64.

Huron, D. (2006). Exploring how music works its wonders. Cerebrum: The Dana Forum on Brain Science, 8(4), 1-6.

Justus, T. \& Hutsler, J. J. (2005). Fundamental issues in the evolutionary psychology of music: Assessing innateness and domain specificity. Music Perception, 23(1), 1-27.

Krumhansl, C. L. (1990). Cognitive Foundations of Musical Pitch. Oxford: Oxford University Press.

Lerdahl, F. \& Jackendoff, R. (1983). A Generative Theory of Tonal Music. Cambridge, MA: MIT Press.

Manzara, L. C., Witten, I. H., \& James, M. (1992). On the entropy of music: An experiment with Bach chorale melodies. Leonardo, 2(1), 81-88.

Narmour, E. (1990). The Analysis and Cognition of Basic Melodic Structures: The Implicationrealisation Model. Chicago: University of Chicago Press.

North, A. C. \& Hargreaves, D. J. (1997). Experimental aesthetics and everyday music listening. In D. J. Hargreaves \& A. C. North (Eds.), The social psychology of music (pp. 84-103). Oxford, UK: Oxford University Press.

Orio, N. (2006). Music retrieval: A tutorial and review. Foundations and Trends in Information Retrieval, $1(1), 1-90$.

Pearce, M. T., Conklin, D., \& Wiggins, G. A. (2005). Methods for combining statistical models of music. In U. K. Wiil (Ed.), Computer Music Modelling and Retrieval (pp. 295-312). Heidelberg, Germany: Springer Verlag.

Pearce, M. T. \& Wiggins, G. A. (2004). Improved methods for statistical modelling of monophonic music. Journal of New Music Research, 33(4), 367-385.

Pinker, S. \& Jackendoff, R. (2005). The faculty of language: What's special about it? Cognition, 95(2), 201-236.

Ritchie, G. (2003). The Linguistic Analysis of Jokes. London/New York: Routledge.

Saffran, J. R., Johnson, E. K., Aslin, R. N., \& Newport, E. L. (1999). Statistical learning of tone sequences by human infants and adults. Cognition, 70(1), 27-52.

Salton, G. (1983). Introduction to modern information retrieval. New York: McGraw-Hill.

Uitdenbogerd, A. L. (2002). Music Information Retrieval Rechnology. Doctoral dissertation, RMIT University of Melbourne, Australia.

von Hippel, P. T. (2002). Melodic-expectation rules as learned heuristics. In C. Stevens, D. Burnham, E. Schubert, \& J. Renwick (Eds.), Proceedings of the Seventh International Conference on Music Perception and Cognition (pp. 315-317). Adelaide, Australia: Causal Productions. 
von Hippel, P. T. \& Huron, D. (2000). Why do skips precede reversals? The effects of tessitura on melodic structure. Music Perception, 18(1), 59-85.

Vos, P. G. \& Troost, J. M. (1989). Ascending and descending melodic intervals: Statistical findings and their perceptual relevance. Music Perception, 6(4), 383-396.

Wiggins, G. A. (2006). A preliminary framework for description, analysis and comparison of creative systems. Journal of Knowledge Based Systems, 19(7), 449-458. 